\title{
AC 2008-145: USING DECISION TREES TO TEACH VALUE OF INFORMATION CONCEPTS
}

\section{Christopher Jablonowski, University of Texas at Austin}

Christopher J. Jablonowski is an Assistant Professor in the Department of Petroleum and Geosystems Engineering, and Associate Director of the Energy and Earth Resources Graduate Program at the University of Texas at Austin. Prior to joining the faculty at UT, he worked as a consultant with Independent Project Analysis, Inc. where he performed empirical research and capital project studies for oil and gas companies worldwide. He has also held positions as a Senior Drilling Engineer and Buyer with Shell Oil Company, and as an Energy Economist with the U.S. Government where he specialized in African and Middle East energy issues and quantitative analysis of energy markets. Dr. Jablonowski earned a B.S. in Civil Engineering from Virginia Tech, a M.B.A. from Tulane University, and a Ph.D. in Energy, Environmental, and Mineral Economics from Penn State. 


\title{
Using Decision Trees to Teach Value of Information Concepts ${ }^{\mathrm{a}}$
}

\begin{abstract}
Most undergraduate engineering economics textbooks and related curricula include elements of decision analysis, and decision trees are often introduced and promoted as a decision making tool. The teaching of value of information analysis is less prevalent, notwithstanding the variety of potential applications to everyday decisions in engineering practice. This paper addresses this gap by providing a detailed demonstration of how decision trees can be used to value information. It includes a detailed set of decision trees that guide the student through a decision under uncertainty. After definition of a base case, cases are provided for the value of perfect and imperfect information. The value of incremental improvement in information is addressed, and a probabilistic approach is described and demonstrated. The influence of risk preference is also addressed.
\end{abstract}

\section{Introduction}

Most undergraduate engineering economics textbooks and related curricula include chapters or modules on decision analysis, and decision trees are often introduced and promoted as a decision making tool. The teaching of value of information (VOI) analysis within the decision analysis frame is less prevalent, notwithstanding the variety of potential applications to everyday decisions in engineering practice. This paper seeks to remedy this gap by providing an accessible demonstration of how decision trees can be used to teach important VOI concepts, including an analysis of the role of risk preferences.

It is often most efficient to set up and solve VOI problems in a generalized analytical framework, and this is often the approach taken in applied research. ${ }^{1-3}$ But analytical representations of VOI problems are not particularly intuitive and can confuse rather than enlighten students. Experience in the classroom has shown that decision trees are a more effective vehicle for teaching VOI concepts. The graphical representation is appealing and students tend to grasp the concepts rather quickly, typically in just one extended lecture.

This paper provides a detailed set of decision trees that guide the student through a decision under uncertainty. After definition of a base case, cases are provided for the value of perfect and imperfect information. The value of incremental improvement in information is addressed, and a probabilistic approach is described and demonstrated. The influence of risk preference is also addressed. The case is targeted at upper level undergraduates in project economics and engineering statistics.

\section{The Base Case}

We first examine a risk-neutral utility maximizing decision-maker with a utility function given by $U(\$ X)=X u$, where $u$ is the unit of utility. ${ }^{\mathrm{b}}$ The base case is depicted in Figure 1. The decision-maker faces a choice between investing in a project or doing nothing. If he invests, the unconditional probability of success is estimated to be 0.15 with a payoff of $\$ 500$. The 
unconditional probability of failure is estimated to be 0.85 with payoff of $-\$ 100$. If he does nothing, the payoff is $\$ 0$. The expected utility of node B is computed as follows:

$E U(B)=0.15 U(\$ 500)+0.85 U(-\$ 100)=0.15(500 u)+0.85(-100 u)=-10 u$. The certainty equivalent of $-10 u$ equals $-\$ 10$. In this case, the decision-maker chooses to do nothing and earns a payoff of $\$ 0$. In the base case, the project has no value.

Next, we consider a case of perfect information. That is, if the decision-maker receives information that perfectly indicates whether the project will be a success or failure, how does this change the decision? This case is depicted in Figure 2. If we assume that the unconditional probabilities of success and failure estimated above are in fact accurate (i.e. they represent the true state of nature), one can compute $E U(B)$ by examining each potential investment decision in isolation. Regardless of what the perfect information indicates, the decision-maker faces the same investment decision. The difference is that the outcome of the invest option is now known with certainty. In the case where the information indicates that the investment will be a success, it is clear that at node A1 the decision will be made to invest, the payoff will be $\$ 500$, and the utility will be $500 u$. In the case where the information indicates that the investment will be a failure, the decision at node $\mathrm{A} 2$ will be made to do nothing, the payoff will be $\$ 0$, and the utility will be $0 u$. Therefore, the expected utility of node B is computed as follows: $E U(B)=0.15(500 u)+0.85(0 u)=75 u$. The certainty equivalent equals $\$ 75$. In this case, the perfect information results in an increase in the expected value of the project. The expected value is the maximum willingness to pay (WTP) for the information.

Perfect information rarely will be available in practice. Instead, decision-makers employ imperfect information. A case of imperfect information is depicted in Figure 3. We assume that performance statistics are available for the source of imperfect information based on results from past performance in similar circumstances. For example, the source predicts success with a probability of 0.60 , and when the source predicts success, he is correct with a probability of 0.20 and incorrect with a probability of 0.80 . The remaining probabilities are defined in Figure 3 . To compute the expected utility of node $\mathrm{D}$ one must examine each potential prediction, again by means of backward induction. The expected utility of node B1 is computed as follows: $E U(B 1)=0.20 U(\$ 500)+0.80 U(-\$ 100)=20 u$. The certainty equivalent equals $\$ 20$ and the decision at node A1 is to invest given a prediction of success. The expected utility of node B2 is computed as follows: $E U(B 2)=0.075 U(\$ 500)+0.925 U(-\$ 100)=-55 u$. The certainty equivalent equals $-\$ 55$ and the decision at node $\mathrm{A} 2$ is to do nothing given a prediction of failure (yielding $0 u$ and $\$ 0$ ). Finally, the expected utility of node $\mathrm{D}$ is computed as follows: $E U(D)=0.60(20 u)+0.40(0 u)=12 u$. The certainty equivalent equals $\$ 12$. This value is the maximum WTP for the imperfect information. The imperfect information is worth less than the perfect information, but still delivers value to the decision-maker. Again, we assume that the unconditional probabilities of success and failure have not changed. ${ }^{\mathrm{c}}$

The structure of Figure 3 enables a clear assessment of the value of incremental improvement in information accuracy. For example, assume the performance statistics for the source of imperfect information improved so that when the source predicts success, he is correct with a probability of 0.30 (not 0.20 ) and incorrect with a probability of 0.70 (not 0.80 ). Assume the conditional probabilities associated with prediction of failure remain constant. First, recognize that when the 
conditional probabilities change, the unconditional probabilities of the two predictions also change because the unconditional probabilities of success and failure are constant. This detail is easy to overlook and will lead to incorrect assessments. The updated structure is depicted in Figure 4 with updated values shaded for emphasis. In this case, one must solve for the new unconditional probabilities of each prediction as follows:

$\operatorname{Pr}($ Success $)=0.15=\operatorname{Pr}($ Prediction $=$ Success $)(0.30)+(1-\operatorname{Pr}($ Prediction $=$ Success $))(0.075) ;$

$\operatorname{Pr}($ Prediction $=$ Success $) \cong 0.33$.

The expected utility of node $\mathrm{B} 1$ is computed as follows: $E U(B 1)=0.30 U(\$ 500)+0.70 U(-\$ 100)=80 u$. The certainty equivalent equals $\$ 80$ and the decision at node $\mathrm{A} 1$ is to invest given a prediction of success. The certainty equivalent at node $\mathrm{B} 2$ has not changed and equals $\$ 0$. The expected utility of node $\mathrm{D}$ is computed as follows: $E U(D)=0.33 U(\$ 80)+0.67 U(\$ 0)=26.67 u$. The certainty equivalent equals $\$ 26.67$. When comparing the two cases of imperfect information, one can compute the change in value attributable to the improvement in accuracy: $\$ 26.67-\$ 12=\$ 14.67$. This figure represents the WTP, or marginal benefit, of the improvement. If the improvement can be obtained for less than this amount, it should be pursued.

\section{Sensitivity Analysis}

A sensitivity analysis on the value of information and the WTP for improvement in information accuracy is readily performed. The results of such an analysis can be compared to the marginal cost of delivering improvement, thus determining the optimal level of investment. ${ }^{3,4}$ When there are multiple cases or uncertainty in the payoffs, the WTP can be evaluated in the same manner. The results of the analysis in this case are depicted in Figure 5. Observe that the positions of the curves in the two cases where the success payoff is less than the base case are higher and lower than the base case. Also, in the case where the success payoff equals $\$ 200$, the initial improvement in accuracy from 0.2 to 0.3 has no value. In performing these computations, one must be careful to account for potential effects in other parts of the decision tree. Although not depicted here, a similar analysis can be performed on node B2 (in isolation, or simultaneously with the analysis on node B1).

\section{Probabilistic Analysis}

This case is also amenable to teaching probabilistic methods for estimating the value of information. The probabilities and/or payoffs can be random variables defined by probability density functions, and simulations can be used to develop probabilistic representations of the value of information. This approach is useful in cases where decisions are being made to invest in improving information accuracy when the potential improvements are not known with certainty. This is often the case in engineering practice Extending the case of imperfect information as depicted in Figure 4, we can examine a case where the decision-maker expects to improve the $\operatorname{Pr}($ Success $\mid$ Prediction $=$ Success $)$ from 0.2 to $X$, where $X \sim N(\mu=0.4, \sigma=0.05)$. These computations can be performed using any software that has random number generation functionality (e.g. MS Excel). Results can be reported using a histogram and cumulative distribution functions. Results for the case described here using 100 simulations are depicted in 
Figure 6. It is also possible to simulate uncertainty in other probabilities, the payoffs, or both. Figure 7 depicts the results of a simulation where, as above, the

$\operatorname{Pr}($ Success $\mid$ Prediction $=$ Success $)$ is expected to improve from 0.2 to $X$, where

$X \sim N(\mu=0.4, \sigma=0.05)$, and the success payoff is a random

variable $\sim N(\mu=\$ 500, \sigma=\$ 100)$. The resulting probability distributions provide decisionmakers with additional information on which to base an investment decision.

It is also helpful for student understanding to discuss the fact that under certain conditions, information may have no value. That is, if the information does not cause the decision-maker to change a decision vis-à-vis the status quo, the WTP is zero. If we again examine Figure 3 and recall the previous calculations, we observe that when the information indicates success the decision is made to invest, and when it indicates failure the decision is made to do nothing. The imperfect information has value because it causes the decision-maker to invest when success is indicated. If we again hold constant the conditional probabilities associated with decision node A2 and examine decision node A1, it is straightforward to compute the probabilities that would make the decision-maker indifferent between investing and doing nothing:

$$
\begin{aligned}
E U(B 1)=0 u= & \operatorname{Pr}(\text { Success } \mid \text { Prediction }=\text { Success }) U(\$ 500)+ \\
& (1-\operatorname{Pr}(\text { Success } \mid \text { Prediction }=\text { Success })) U(-\$ 100) .
\end{aligned}
$$

Solving for the unknown probability, $\operatorname{Pr}($ Success $\mid$ Prediction $=$ Success $)=1 / 6$. Therefore, when this probability is less than $1 / 6$, the decision is made to do nothing, and the information has no value. The concept of an information value threshold is an important learning objective. A common intuition among students is that information always adds value, and this simple example clearly demonstrates that this is not always the case. Information can only have value when it changes a decision. ${ }^{5}$

\section{Accounting for Risk Preferences}

The identical framework is employed to assess the value of information for a non-risk neutral decision maker. Here, we depict a case where the decision maker is risk averse. The decision maker's utility function is defined as follows: $U(\$ X)=75+1.6 X-0.0015 X^{2} u$, for $-100 \leq X \leq 500 .{ }^{\mathrm{d}}$ The following results will be required in several places below: $U(\$ 500)=500 u ; U(\$ 0)=75 u ; U(-\$ 100)=-100 u$. Referring to the base case of Figure 1 , the expected utility of node $\mathrm{B}, E U(B)$, is computed as follows: $E U(B)=0.15 U(\$ 500)+0.85 U(-\$ 100)=0.15(500 u)+0.85(-100 u)=-15 u$.

The certainty equivalent of $-15 u$ equals $-\$ 53.56$ (recall, $-100 \leq X \leq 500$, so this is the relevant root). Therefore, the decision-maker chooses to do nothing and earns a payoff of $\$ 0$.

In the perfect information case of Figure 2, the expected utility of node $\mathrm{B}$ is computed as follows: $E U(B)=0.15 U(\$ 500)+0.85 U(\$ 0)=0.15(500 u)+0.85(75 u)=138.75 u$. The certainty equivalent equals $\$ 41.45$. In this case, the perfect information results in decision-maker response (investing when success is indicated) and a positive value of the perfect information. 
The case of imperfect information is again depicted in Figure 3. The expected utility of node B1 is computed as follows:

$E U(B 1)=0.20 U(\$ 500)+0.80 U(-\$ 100)=0.20(500 u)+0.80(-100 u)=20 u$.

The certainty equivalent equals $-\$ 33.33$. Therefore, the decision at node $\mathrm{A} 1$ is to do nothing given a prediction of success. The expected utility of node $\mathrm{B} 2$ is computed as follows: $E U(B 2)=0.075 U(\$ 500)+0.925 U(-\$ 100)=0.075(500 u)+0.925(-100 u)=-55 u$. The certainty equivalent is $-\$ 75.86$, and the decision at node $\mathrm{A} 2$ is to do nothing given a prediction of failure. The investment decision is not conditional on the information and therefore the imperfect information has no value. This result contrasts with the risk-neutral outcome where the value of imperfect information was positive. This result demonstrates that information value is a function of risk preferences, and that making the simplifying assumption of risk-neutrality can lead to erroneous estimates. Also, while not demonstrated here, the degree of risk aversion will also affect information value, although the relationship is not necessarily monotonic. ${ }^{9}$

The sensitivity analysis and probabilistic analysis demonstrated above can be repeated for the case of risk aversion, although this is not reported here. Also, it is possible to compute the threshold for positive information value. If we again hold constant the conditional probabilities associated with decision node $\mathrm{A} 2$ and examine decision node $\mathrm{A} 1$, it is straightforward to compute the probabilities that would make the decision-maker indifferent between investing and doing nothing (recall, doing nothing yields $\$ 0$ and $75 u$ ):

$$
\begin{aligned}
E U(B 1)=75 u= & \operatorname{Pr}(\text { Success } \mid \text { Prediction }=\text { Success }) U(\$ 500)+ \\
& (1-\operatorname{Pr}(\text { Success } \mid \text { Prediction }=\text { Success })) U(-\$ 100) .
\end{aligned}
$$

Solving for the unknown probability, $\operatorname{Pr}($ Success $\mid$ Prediction $=$ Success $)=0.29$. Unless this probability exceeds this value, the decision remains to do nothing, and the information has no value.

\section{Conclusion}

Experience in the classroom has demonstrated that decision trees are a more effective vehicle for introducing VOI concepts than analytical presentations. This paper provides a detailed set of decision trees that guide the student through a decision under uncertainty. The following key teaching points were emphasized:

- Information can only have value when it changes a decision;

- Sensitivity and probabilistic analyses on the WTP for information can inform optimal investment decisions regarding information improvement;

- Risk preferences influence the value of information;

- The degree of risk aversion affects information value, although the direction of the effect is specific to the utility function and nature of the payoffs and probabilities.

Potential applications of VOI analyses in engineering practice are widespread, although they are not always be recognized as such. There are obvious applications in the geosciences, mining and petroleum engineering, and geotechnical engineering. An example of a less obvious application is in R\&D. For example, research can be initiated to improve the accuracy and/or precision of a 
measurement, but one should also estimate whether or not such improvements actually increase value vis-à-vis decisions based on the subject measurement. Ignoring such calculations can lead to over- and underinvestment in information improvement. When students are sensitized to VOI thinking early in their education, they will examine such investments more critically in their coursework and as practicing engineers.

\section{References}

1. Katz, R., and A. Murphy. 1997. Economic Value of Weather and Climate Forecasts. Cambridge University Press: Cambridge, UK.

2. Granger, C. and M. Pesaran. 2000. Economic and Statistical Measures of Forecast Accuracy. Journal of Forecasting, 19: 537-560.

3. Considine, T., Jablonowski, C., Posner, B., and C. Bishop. 2004. The Value of Hurricane Forecasts to Oil and Gas Producers in the Gulf of Mexico. Journal of Applied Meteorology: 43 (9): 328-336.

4. Jablonowski, C. and R. MacAskie. 2007. The Value of Oil and Gas Price Forecasts. Proceedings of the SPE Hydrocarbon Economics and Evaluation Symposium, Dallas, Texas, April 1-3.

5. Bratvold, R., Bickel, J. and H. Lohne. 2007. Value of Information in the Oil and Gas Industry: Past, Present, and Future. Proceedings of the SPE Annual Technical Conference and Exhibition, Anaheim, CA, November 11-14.

6. Cicchetti, C., and J. Dubin. 1994. A Microeconometric Analysis of Risk Aversion and the Decision to Self Insure. Journal Of Political Economy, 102 (1): 169-186.

7. Walls, M. and J. Dyer. 1996. Risk Propensity and Firm Performance: A Study of the Petroleum Exploration Industry. Management Science, 42 (4): 1004 - 1021.

8. Jablonowski, C. 2007. Assessing Risk Preferences in E\&P Operational Settings. Energy Exploration and Exploitation, 25 (2): 83-93.

9. Eeckhoudt, L. and P. Godfroid. 2000. Risk Aversion and the Value of Information. Journal of Economic Education, 31 (4): 382-388. 
Figure 1. Base Case

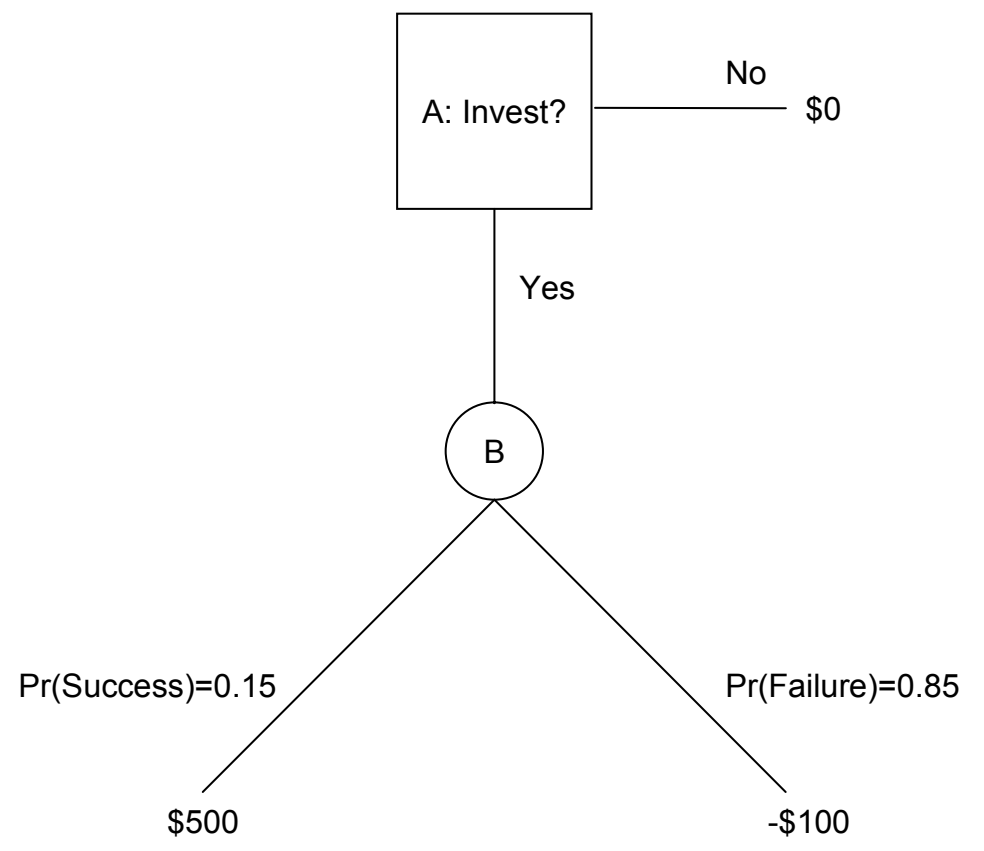

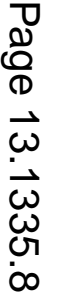


Figure 2. Willingness to Pay for Perfect Information

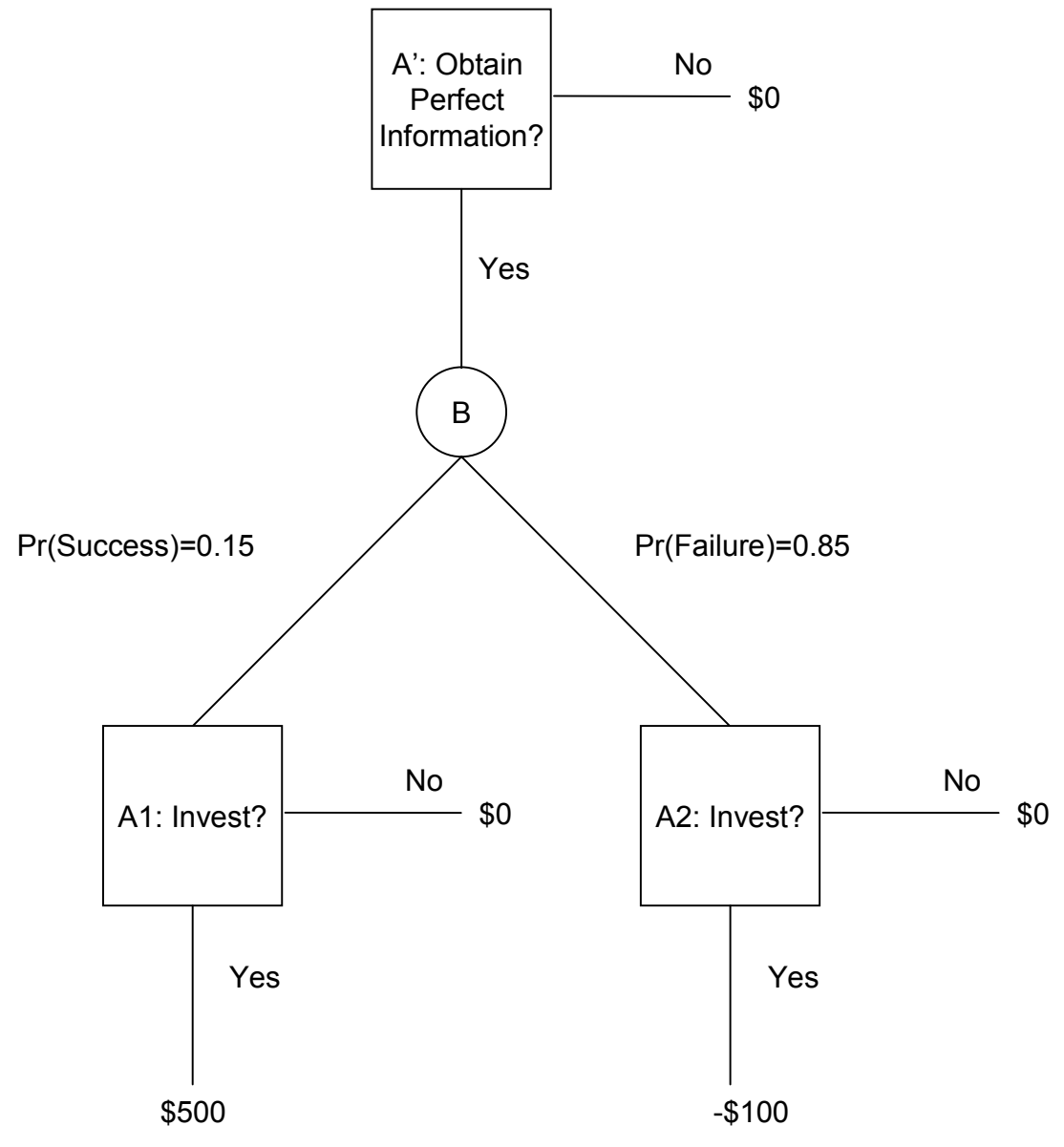


Figure 3. Willingness to Pay for Imperfect Information

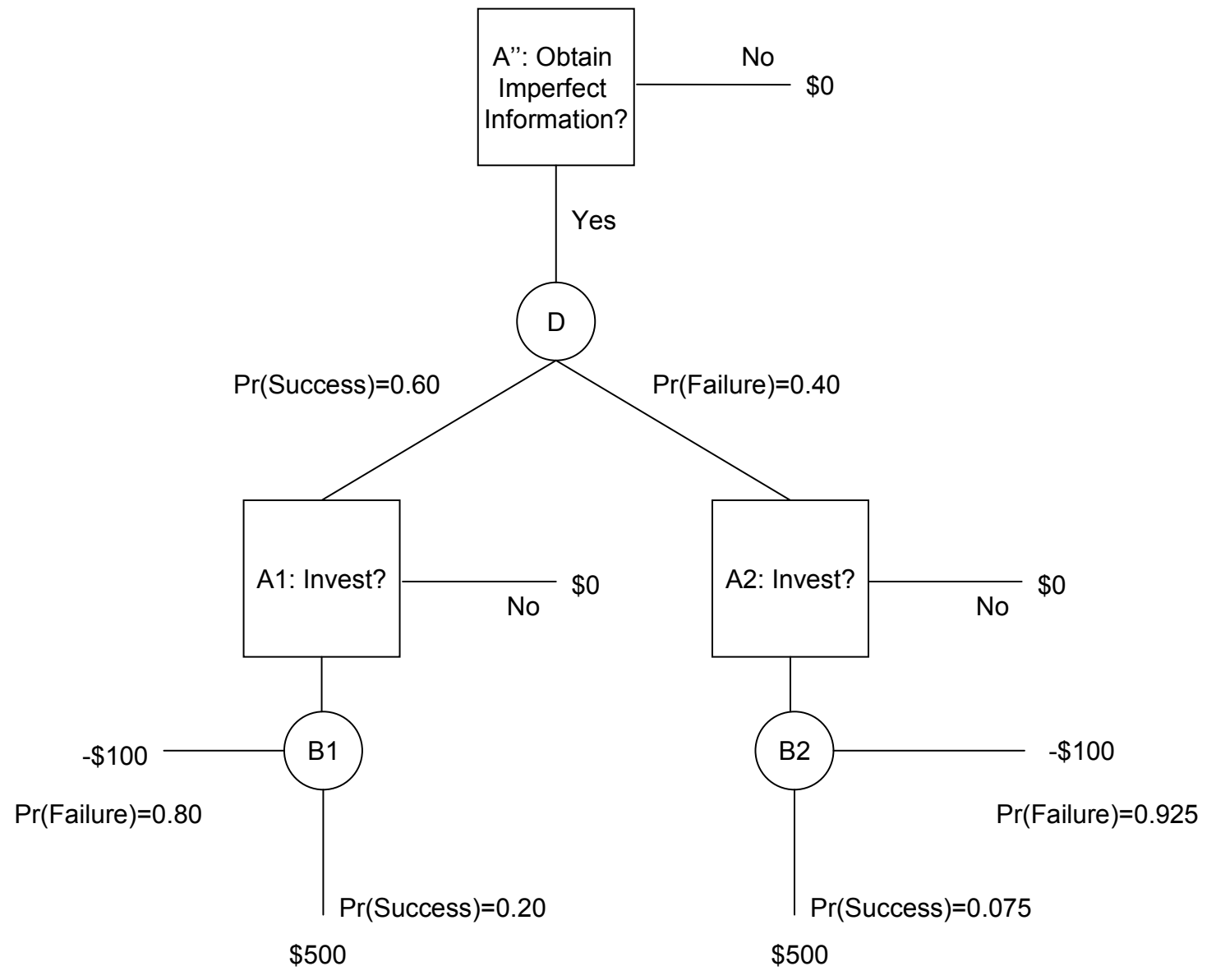


Figure 4. Willingness to Pay for Improvements to Imperfect Information

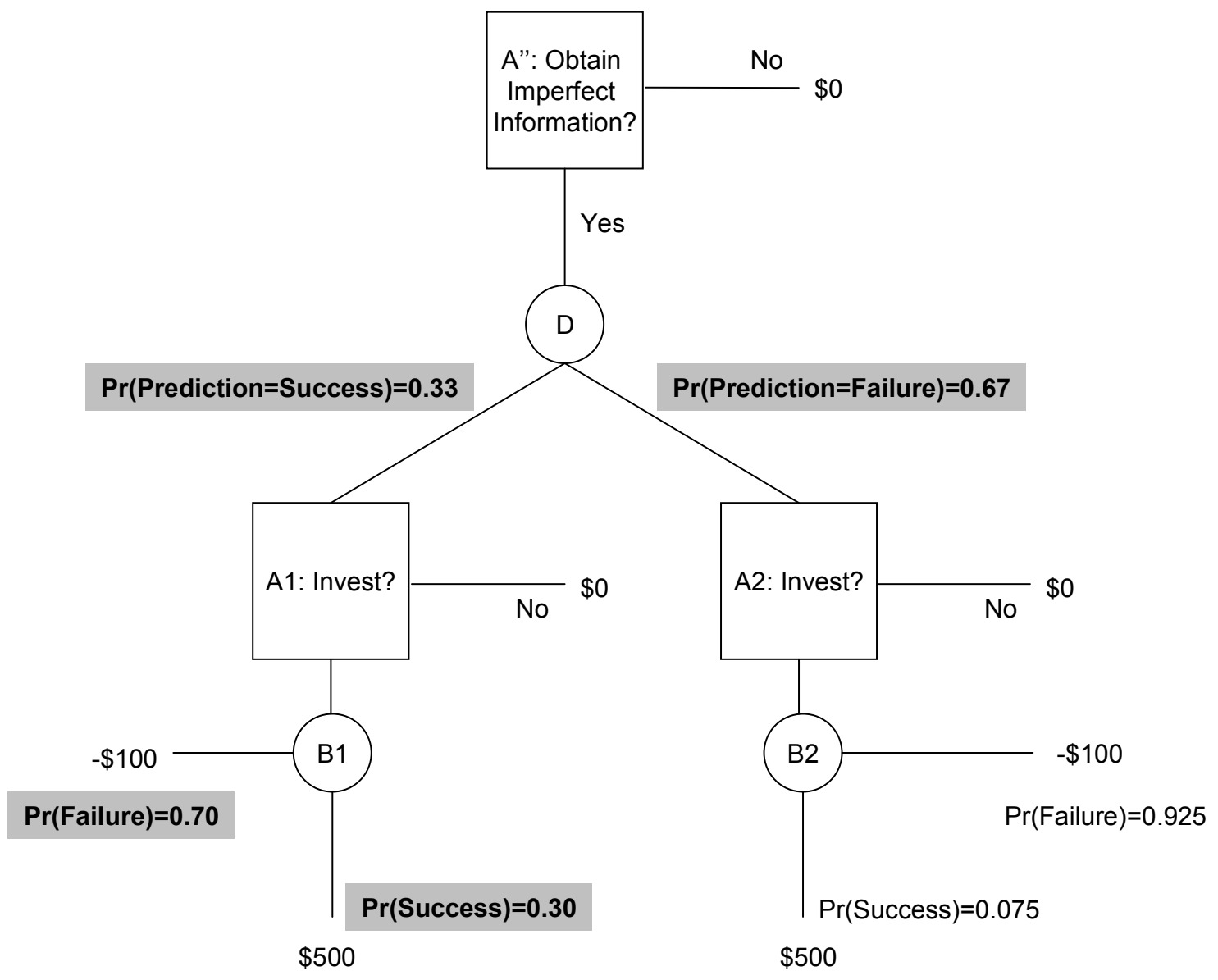


Figure 5. The Sensitivity of Willingness to Pay for Improvement to Information: $\operatorname{Pr}($ Success $\mid$ Prediction $=$ Success $)$ at Node B1

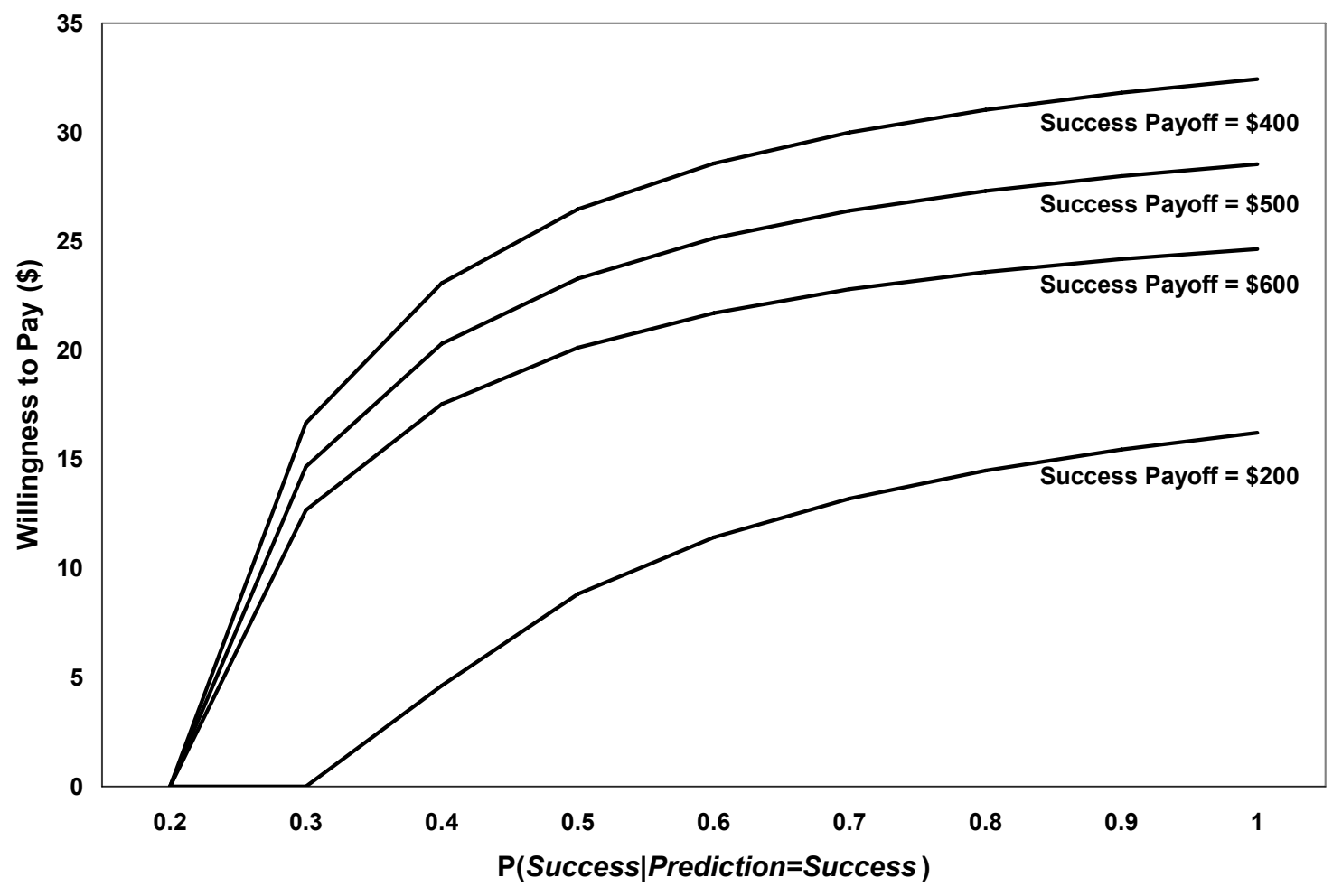


Figure 6. Probabilistic Willingness to Pay for Improvements to Information:

$\operatorname{Pr}($ Success $\mid$ Prediction $=$ Success) at Node B1 (Uncertainty in the Conditional Probability)

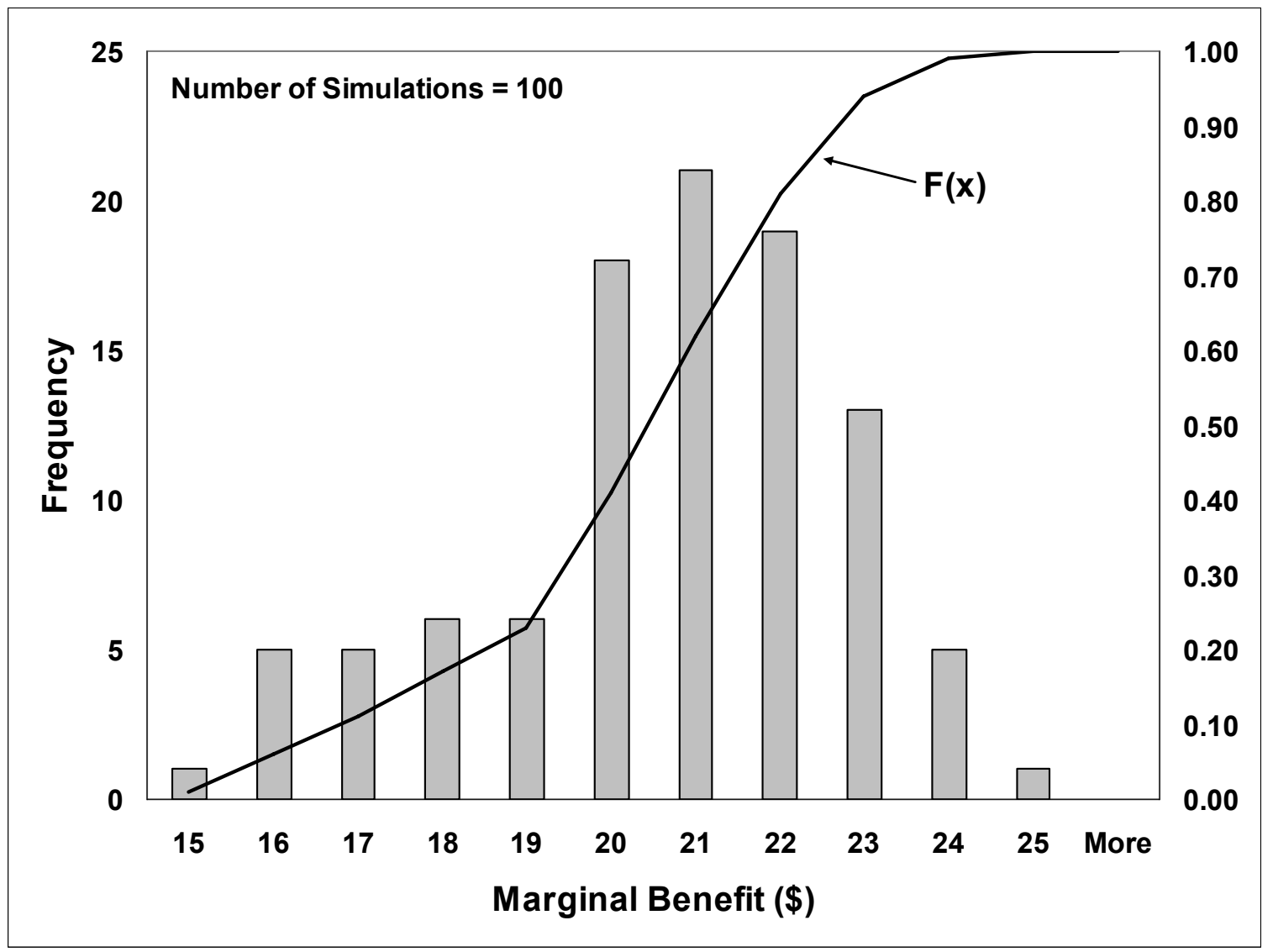


Figure 7. Probabilistic Willingness to Pay for Improvements to Information: $\operatorname{Pr}($ Success|Prediction $=$ Success) at Node B1 (Uncertainty in the Conditional Probability and the Success Payoff)

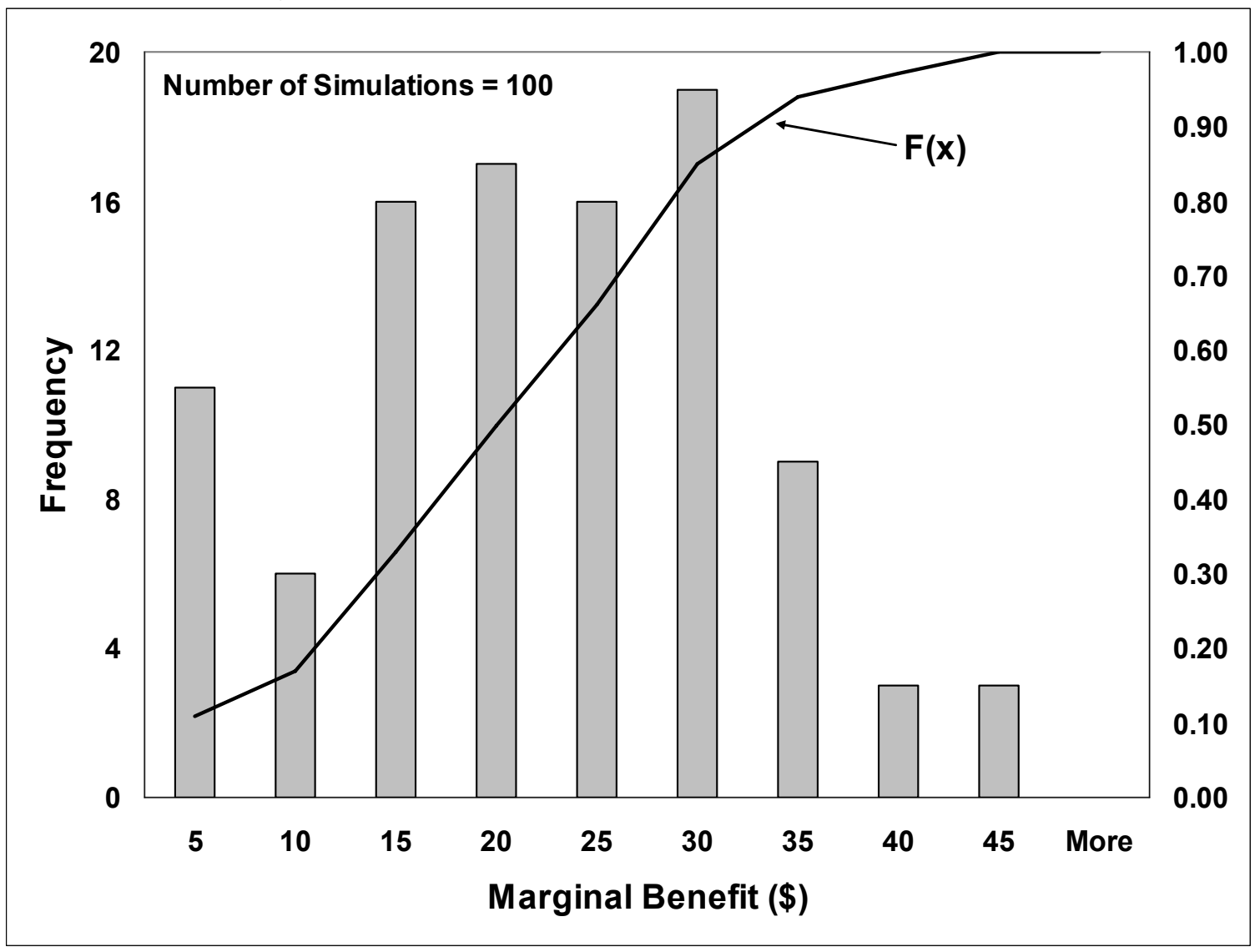

${ }^{\mathrm{a}}$ The author thanks the referees and students in my undergraduate project analysis and geostatistics courses for constructive comments. The author assumes sole responsibility for errors and omissions, and the views expressed herein. Electronic versions of this document and the figures are available from the author upon request.

${ }^{\mathrm{b}}$ A risk-neutral utility function is defined by constant marginal utility.

c This can be verified by computing the unconditional probability of success as follows:

$\operatorname{Pr}($ Success $)=0.15=\operatorname{Pr}($ Prediction $=$ Success $) \operatorname{Pr}($ Success $\mid$ Prediction $=$ Success $)+$

$\operatorname{Pr}($ Prediction $=$ Failure $) \operatorname{Pr}($ Success $\mid$ Prediction $=$ Failure $)=0.60(0.20)+0.40(0.075)=0.15$.

${ }^{d}$ This utility function satisfies requirements for risk aversion in the interval specified, namely $d U / d X>0$ and $d^{2} U / d X^{2}<0$, but otherwise it is arbitrary. There is a literature on the empirical estimation of utility functions. ${ }^{6-8}$ 